\title{
Diferencia de los niveles de inteligencia emocional-social entre los estudiantes católicos practicantes y no practicantes de un colegioprivado
}

\author{
Difference of levels in emotional-social intelligence among practicing and \\ non-practicing catholics students of a private school
}

\author{
SANDRAANCHORENA O ${ }^{1}$ \\ Universidad Nacional Mayor de San Marcos, Lima, Perú \\ (RECIBIDO 05-09 2014, ACEPTADO 16-11-2014)
}

\begin{abstract}
RESUMEN
Esta investigación comparó la diferencia en los niveles de inteligencia emocional-social entre los estudiantes católicos practicantes y no practicantes de un colegio católico privado del distrito de Surco, en Lima Metropolitana. La muestra de 148 adolescentes de cuarto y quinto de secundaria, de ambos sexos, de 14 a 17 años, se dividió en dos grupos según su frecuencia de asistencia a misa. Se utilizó el inventario de cociente emocional para adolescentes (EQ-i:YV) de Bar-On, adaptado a la población peruana. Los estudiantes católicos practicantes obtuvieron mejores puntajes en la pruebay en todas sus dimensiones o subescalas. La prueba t de Student demostró diferencias significativas en inteligencia emocional-social y en dos de sus dimensiones: interpersonal y manejo del estrés, a favor de los estudiantes católicos practicantes.
\end{abstract}

Palabras clave: Espiritualidad, católico, misa, inteligencia emocional-social.

\begin{abstract}
This study compared the difference in levels of emotional-social intelligence among practicing and non-practicing catholic students of a private catholic school in the district of Surco in Lima. The sample of 148 adolescents of fourth and fifth secondary grade of both sexes aged 14 to 17 years old, it was divided in two groups according to frequency of Mass attendance. The Bar-On Emotional Quotient Inventory Young Version (EQ-i:YV) adapted to the Peruvian population, it was used. Practicing catholic students scored better on the test and in all its dimensions or sub-scales. The Student $t$ test showed significant differences in emotionalsocial intelligence and in two of its dimensions: interpersonal and stress management, where practicing catholic students outscored the non-practicing catholic students.
\end{abstract}

Keywords: Spirituality, catholic, Mass, emotional-social intelligence.

1 Egresada de maestría en Psicología Educativa, UNMSM. E-mail: csandrap@yahoo.com 


\section{INTRODUCCIÓN}

En un mundo tan competitivo como el actual, ya no bastan los grados académicos para alcanzar el éxito profesional y menos aún para alcanzar lo más importante y que incluye el anterior: el éxito personal integral, es decir, llegar a ser humano, desarrollado y feliz.

Primero se pensó que llegaríamos a este tan ansiado éxito personal integral desarrollando nuestra inteligencia emocional, la cual demostró ser tan importante como la inteligencia cognitiva o racional. Pero no fue suficiente.

Es entonces que surge la respuesta y observamos en el mundo un interés redescubierto hacia la vida espiritual. El hombre vuelve la mirada hacia lo que un día conoció y que luego dejó de lado y que acaso sea la razón por la que ahora se ve envuelto en tanta violencia e infelicidad.

Es por esto que en el mundo se está empezando a reconocer la gran importancia y el gran impacto que tiene la vida espiritual para el ser humano y su pleno desarrollo personal.

La espiritualidad (últimamente llamada inteligencia espiritual) comienza a ser tomada en cuenta por personas que en el mundo actual han alcanzado el éxito profesional y personal y que son célebres conocedores de psicología como Goleman (2012) y Fischman (2014).

Hoy, el término inteligencia espiritual empieza a aparecer en los negocios. Gurús como Peter Senge, entre otros, lo mencionan en sus libros. Prestigiosas universidades americanas ofrecen cursos de pregrado y posgrado de liderazgo y espiritualidad y de inteligencia espiritual (Fischman, 2014).

Finalmente, las personas con inteligencia espiritual entienden y sienten que todos somos parte de un gran todo. Comprenden que hacer daño a otro es también hacerse daño a uno mismo y que ayudar nos da una felicidad profunda. En el plano espiritual todos estamos conectados (Fischman, 2014).

Silas personas tuvieran más inteligencia espiritual se acabarían los conflictos laborales, los problemas interpersonales, la competencia interna. Además, se serviría al cliente no por la función sino porque le nacería a las personas. En el mundo se acabaría la pobreza extrema, las guerras y reinaría la paz (Fischman, 2014).

El camino de buscar la inteligencia espiritual es largo, poco emocionante y requiere mucha perseverancia. Pero es un camino que, además de hacernos más felices, nos permitirá ayudar a este mundo a mejorar (Fischman, 2014).

En esta investigación se ha querido demostrar cómo la vida espiritual o espiritualidad influye directa o indirectamente en todas las demás áreas de la vida del ser humano, para bien o para mal según qué espiritualidad se escoja seguir, y si somos coherentes con nuestra fe.

Para medir la espiritualidad se ha tomado como variable controlada la frecuencia de asistencia a Misa de los estudiantes de la muestra, dividiéndolos en católicos practicantes y católicos no practicantes. 
Luego se hace una comparación entre los niveles de inteligencia emocional-social obtenidos por los estudiantes católicos practicantes y no practicantes.

\section{Objetivo}

Identificar las diferencias que existen entre los estudiantes católicos practicantes y no practicantes de un colegio privado del distrito de Surco, con respecto a la inteligencia emocional-social y a sus dimensiones.

\section{Misa}

Ceremonia religiosa de la Iglesia Católica en la que se celebra el sacramento de la Eucaristía y se hace el memorial de la muerte y la resurrección de Jesús. Consta de una primera parte en la que se leen las lecturas bíblicas del día y el sacerdote hace la homilía y una segunda parte en la que tiene lugar la consagración del pan y del vino y se reparte la comunión.

Los que conscientemente faltan a la misa cometen pecado grave, porque le niegan el culto debido a Dios y rechazan su gracia (Catecismo Menor, 2012).

Para los católicos es obligatorio asistir a misa todos los domingos y feriados religiosos.

La asistencia a misa es una práctica fundamental y propia de la religión católica. Al considerarla en esta investigación, hemos querido medir sus efectos en los asistentes y compararlos con los que no asisten o asisten con poca frecuencia; para esto hemos dividido la muestra entre católicos practicantes y católicos no practicantes respectivamente.

\section{Homilía}

Discurso o sermón sencillo que pronuncia en público un sacerdote y que contiene explicaciones o instrucciones sobre ciertas materias religiosas, a menudo relacionadas con la sociedad o la actualidad.

\section{La fe}

Cabe indicar que si una persona decide asistir a misa todos los domingos y fiestas religiosas, en el mejor de los casos lo hará porque cree en Dios, es decir, porque tiene fe.

La psicología ha intentado estudiar a la fe, sin lograrlo; se ha acercado llegando a estudiar la religión en psicología de la religión. Pero la fe no la podemos estudiar dentro del mundo natural o del campo de la ciencia porque no la podemos producir humanamente ya que es un don sobrenatural (es decir, un regalo de Dios). Por esto, si queremos saber sobre la fe, debemos basarnos en fuentes creyentes, es decir, personas que tienen vida espiritual, que tienen comunicación con Dios 
por medio de la oración constante, con autoridad moral dada por el mismo Dios como es el caso de los papas.

"No se contenten con una religiosidad exterior. A Dios no le basta que su pueblo lo venere con los labios; quiere nuestro corazón y nos da su gracia si no nos alejamos o separamos de ÉI" (S.S. Benedicto XVI, discurso a los obispos de Austria en visita ad limina, 5 de noviembre, 2005).

\section{Las inteligenciasmúltiples}

En 1983, Gardner escribió un libro titulado Frames of Mind [Estructuras de la mente en donde expuso su teoría acerca de la inteligencia humana. Su objetivo era lograr un enfoque del pensamiento humano que fuera más amplio y completo que el que podía derivarse de los estudios cognitivos realizados hasta el momento. Gardner propone dejar de hablar de inteligencia y considerar el concepto de "inteligencias múltiples". Utiliza el concepto de "múltiples" con el objetivo de resaltar el número desconocido de capacidades humanas, desde la inteligencia musical hasta la inteligencia aplicada en el conocimiento de uno mismo, que no han sido tomadas en cuenta en el estudio tradicional de la inteligencia. Con el sustantivo "inteligencias", Gardner resalta que estas capacidades son tan fundamentales como las que tradicionalmente detecta el test de $\mathrm{Cl}$. Su teoría acaba con la veracidad de la afirmación que en 1923 llevó a cabo Boring cuando definió la inteligencia como "lo que miden esos tests". Desde su concepción de inteligencia, lo que miden esos tests no es inteligencia, sino solo una parte de ella (adaptado de Molero, Saíz \& Esteban, 1998).

Gardner $(1983,1999)$ se ha rebelado contra una concepción monolítica y estable de la inteligencia, el desafío para él no se trata sólo de saber qué es la inteligencia o cómo la podemos desarrollar, sino de saber combinar la inteligencia y la ética para crear un mundo en el que todos queramos y podamos vivir. Después de todo, una sociedad dirigida por personas inteligentes bien podría saltar por los aires o acabar con todo el planeta. La inteligencia es valiosa, pero la personalidad es más importante (Gardner, 1999; citado por Pérez \& Beltrán, 2006).

La teoría de las inteligencias múltiples (IM) de Gardner (1983) ha contribuido, junto con otras, a cambiar los puntos de vista tradicionales sobre la inteligencia humana excesivamente centrados en el $\mathrm{Cl}$, abriendo nuevos espacios a la intervención psicoeducativa, con la esperanza de ofrecer una educación de calidad y, sobre todo, de mejorar el funcionamiento cognitivo de los alumnos (Pérez \& Beltrán, 2006).

Gardner $(1983,1999)$ afirma que hay muchas maneras de ser inteligente, como estas:

1) Inteligencia lingüística: Se trata de la inteligencia relacionada con la capacidad verbal, el lenguaje y las palabras. Se refiere a la adecuada construcción de las oraciones, la utilización de las palabras de acuerdo con sus significados y sonidos, al igual que la utilización del lenguaje de conformidad con sus diversos usos. 
2) Inteligencia lógico-matemática: Se relaciona con el pensamiento abstracto, centrado en la precisión y organización mediante pautas o secuencias. Referida a la facilidad para manejar cadenas de razonamiento e identificar patrones de funcionamiento.

3) Inteligencia espacial o visual: Se refiere a la habilidad para integrar elementos, percibirlos y ordenarlos en el espacio, estableciendo a continuación relaciones metafóricas entre ellos, y a la capacidad de visualizar objetos desde perspectivas diferentes.

4) Inteligencia kinestésica-corporal: Señala la capacidad para manejar el cuerpo en la realización de movimientos en función del espacio físico así como manipular objetos con destreza.

5) Inteligencia musical: Se refiere al uso adecuado del ritmo, melodía y tono en la construcción y apreciación musical.

6) Inteligencia interpersonal: Habilidad y capacidad para establecer relaciones con otros individuos, también para reconocer las emociones y sentimientos derivados de las relaciones entre las personas y sus grupos.

7) Inteligencia intrapersonal: Señala la capacidad que tiene una persona para conocer su mundo interno, es decir, sus propias y más íntimas emociones y sentimientos, así como sus propias fortalezas y debilidades.

8) Inteligencia naturalística: Se refiere a la habilidad para discriminar y clasificar los organismos vivos existentes en la naturaleza. Estas personas se reconocen parte del ecosistema ambiental.

9) Inteligencia existencial: últimamente admitida por el autor y que él define como la inteligencia de las grandes preguntas (Gardner 2005b). Señala la sensibilidad por la existencia del ser humano, por reflexiones sobre la trascendencia humana.

De las variedades de inteligencia propuestas por Gardner, nos interesa destacar las dos formas de inteligencia personal. La inteligencia interpersonal definida como la capacidad para entender a las otras personas: lo que les motiva, cómo trabajar con ellos de forma cooperativa, etc.; y la inteligencia intrapersonal definida como la capacidad correlativa, pero orientada hacia dentro. Es decir, la capacidad de formarse un modelo ajustado, verídico de uno mismo y de ser capaz de usar este modelo para desenvolverse eficazmente en la vida (Gardner, 1983). El motivo por el que resaltamos estos dos tipos de inteligencia es porque ambos han contribuido de forma importante a un nuevo concepto dentro del marco teórico general acerca de la inteligencia, que en el año 1990 fue denominado por Mayer y Salovey como inteligencia emocional (Molero et al., 1998).

\section{La inteligencia emocional}

El término "inteligencia emocional" fue acuñado por Salovey y Mayer (1990, citados por Ugarriza, 2001) y definido por estos autores como un tipo de inteligencia social que engloba la habilidad para dirigir y controlar nuestras propias emociones y las de los demás, así como para discriminar entre ellas y utilizar la 
información que nos proporciona para guiar nuestro pensamiento y acciones, de tal forma que resulten beneficiosas para nosotros mismos y para la cultura a la que pertenecemos.

\section{El modelo deGoleman}

Goleman en 1996 presentó su definición de inteligencia emocional, basándose en los trabajos desarrollados por Mayer y Salovey en 1993, sin embargo agregó muchas cosas que él presentó como inteligencia emocional, como es el caso de la inclusión de variables que podrían considerarse más como "rasgos de personalidad", que como componentes de inteligencia emocional; así, por ejemplo, incluyó el optimismo, la perseverancia y la habilidad de retardar la satisfacción como aspectos relevantes de la inteligencia emocional (Escurra \& Delgado, 2001).

El concepto de inteligencia emocional surge como un intento de responder a esta cuestión, es decir, resaltar el papel que tienen las emociones en nuestra vida intelectual y nuestra adaptación social y equilibrio personal. Para Goleman, el concepto de inteligencia emocional es importante porque, entre otras cosas, constituye el vínculo entre los sentimientos, el carácter y los impulsos morales (Molero et al., 1998).

\section{La inteligencia emocional-social}

Bar-On utiliza el término "inteligencia emocional y social" para denominar un tipo específico de inteligencia que difiere de la inteligencia cognitiva. El autor opina que los componentes factoriales de la inteligencia no cognitiva se asemejan a los factores de la personalidad, pero, a diferencia de ellos, la inteligencia emocional y social puede modificarse a través de la vida (Ugarriza, 2001). Bar-On (2006) define la inteligencia emocional-social, como un conjunto de habilidades personales, emocionales y sociales y de destrezas que influyen en nuestra habilidad para adaptarnos y enfrentar las demandas y presiones del medio.

La persona emocionalmente inteligente es, por lo general, optimista, flexible, realista y exitosa a la hora de resolver problemas y hacer frente al estrés, sin perder el control (Bar-On, 1997; citado por Ferrándiz, Hernández, Bermejo, Ferrando \& Sáinz, 2012).

\section{El inventario del cociente emocional de Bar-On (EQ-i)}

El modelo de Bar-On proporciona la base teórica para el EQ-i, que fue desarrollado originalmente para evaluar diversos aspectos del constructo Inteligencia emocional-social, así como para examinar su conceptualización. Según este modelo, la inteligencia emocional-social (IES) es un conjunto interrelacionado de competencias, habilidades y facilitadores emocionales y sociales que determinan cómo efectivamente nos entendemos y expresamos, comprendemos a otros y nos relacionamos con ellos, y hacemos frente a las demandas diarias (Bar-On, 2006). 
El autor propone 15 subescalas para definir la IES. De estas, diez son consideradas como capacidades básicas y se refieren a aspectos esenciales de la IES (autoconciencia emocional, autoconsideración, asertividad, empatía, relaciones interpersonales, tolerancia al estrés, control del impulso, validación, flexibilidad y solución de problemas); y cinco son consideradas como capacidades facilitadoras de la IES (optimismo, autorrealización, alegría, independencia emocional y responsabilidad social). Estas quince subescalas se organizan en cinco grandes dimensiones para el estudio de las características emocionales y sociales de los sujetos: intrapersonal (habilidad para comprender las propias emociones y su comunicación a los otros); interpersonal (habilidad para entender y apreciar las emociones de los otros); manejo de las emociones (habilidad para dirigir y controlar las propias emociones); adaptabilidad (flexibilidad y eficacia para resolver conflictos) y escala de estado de ánimo general (habilidad para tener una actitud positiva ante la vida). Se trata de un inventario amplio que proporciona información acerca de las competencias emocionales y sociales y que permite trazar un perfil social y afectivo (Bar-On, 1997; Bar-On y Parker, 2000; citados por Férrandiz et al., 2012).

\section{Hipótesis}

Existen diferencias significativas entre los estudiantes católicos practicantes y no practicantes de un colegio privado del distrito de Surco, con respecto a la inteligencia emocional-social (IES) y a sus dimensiones.

\section{MÉTODO}

\section{Tipo y diseño de investigación}

Esta investigación es no experimental, cuantitativa, transversal, descriptiva comparativa, pues busca comparar los niveles de la variable inteligencia emocionalsocial en un momento determinado entre los estudiantes católicos practicantes y los no practicantes de cuarto y quinto de secundaria en un colegio católico privado.

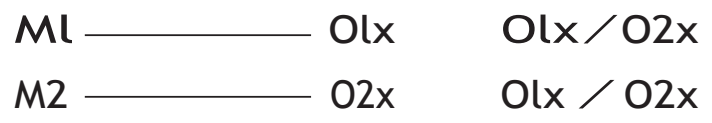

\section{Donde:}

$M:$ Muestra de investigación ( $M=M 1+M 2)$

M1: Estudiantes católicos practicantes. Para efectos de esta investigación, se considera como miembro de este grupo al estudiante católico que asiste a misa todos los domingos y fiestas religiosas o todos los domingos ó una o dos veces al mes o cada dos meses.

M2: Estudiantes católicos no practicantes. Para efectos de esta investigación se considera como miembro de este grupo al estudiante católico que asiste a misa cada tres meses o una o dos veces al año. 
01x, 02x: Observaciones de la variable inteligencia emocional-social (x) en cada una de las submuestras.

\section{Tamaño y selección de la muestra}

Todos los estudiantes de cuarto y quinto de secundaria, varones y mujeres, con edades comprendidas entre 14 y 17 años de un colegio católico privado del distrito de Surco, en Lima Metropolitana, matriculados en el año 2013, entre los cuales se utilizó el criterio de exclusión de pertenecer a otras religiones, sectas o ser ateos. Como resultado el tamaño de la muestra fue de 148 estudiantes, de los cuales 127 fueron considerados católicos practicantes y 21 no practicantes, de acuerdo a su frecuencia de asistencia a misa.

\section{Instrumento}

Se empleó el inventario del cociente emocional versión joven (EQ-i:YV) de BarOn (2000), adaptado a la población peruana por Ugarriza y Pajares (2005), el cual consiste en un cuestionario para la valoración de la inteligencia emocional-social (IES) basado en la versión de adultos. Consta de 60 ítems en su forma completa (es la que se utilizó en este estudio) para evaluar las cinco grandes dimensiones de la IES en una escala Likert de 4 puntos ( $1=$ casi nunca me pasa, $4=$ casi siempre me pasa). EI EQ-i:YV es aplicable a individuos de 8 a 18 años de edad y toma aproximadamente 15 minutos completarlo (Bar-On \& Parker, 2000; citados por Bar-On, 2006).

Se empleó el paquete estadístico SPSS versión 20 para procesar los datos.

\section{Confiabilidad}

Se obtuvo una fiabilidad por coeficiente de alfa de Cronbach de 0.9 para el cociente de inteligencia emocional-social Total. En las subescalas los coeficientes de fiabilidad fueron los siguientes: intrapersonal 0.81, interpersonal 0.76, adaptabilidad 0.84 , manejo del estrés 0.88 , estado de ánimo general 0.89 .

\section{Validez}

Se estableció por la validez de constructo, la cual se comprobó a través delanálisis factorial: el primer factor (estado de ánimo general) explica el $16.91 \%$ de la varianza, el segundo factor (Interpersonal) explica el $7.56 \%$ de la varianza, el tercer factor (manejo del estrés) explica el 6.56\% de la varianza, el cuarto factor (adaptabilidad) explica el $5.73 \%$ de la varianza y el quinto factor (intrapersonal) explica el $5.04 \%$ de la varianza. El conjunto de factores explicaron el $41.8 \%$ de la varianza.

El método de extracción utilizado fue el análisis de componentes principales. 


\section{RESULTADOS}

La Tabla 1 muestra las cantidades tanto en números como en porcentajes de los estudiantes divididos en dos grupos, católico practicante y no practicante, ubicados en los niveles de inteligencia emocional-social alcanzados.

Tabla 1. Niveles de inteligencia emocional-social según frecuencia de asistencia a misa.

\begin{tabular}{|c|c|c|c|}
\hline \multirow[t]{2}{*}{ Inteligencia emocional-social } & \multicolumn{2}{|c|}{ Frecuencia de asistencia a Misa } & \multirow[t]{2}{*}{ Total } \\
\hline & $\begin{array}{c}\text { Católico } \\
\text { practicante }\end{array}$ & $\begin{array}{l}\text { Católico NO } \\
\text { practicante }\end{array}$ & \\
\hline \multirow{2}{*}{ Baja } & 1 & 1 & 2 \\
\hline & $0.8 \%$ & $4.8 \%$ & $1.4 \%$ \\
\hline \multirow{2}{*}{ Adecuada } & 35 & 10 & 45 \\
\hline & $27.6 \%$ & $47.6 \%$ & $30.4 \%$ \\
\hline \multirow{2}{*}{ Alta } & 33 & 5 & 38 \\
\hline & $26.0 \%$ & $23.8 \%$ & $25.7 \%$ \\
\hline \multirow{2}{*}{ Muy alta } & 39 & 4 & 43 \\
\hline & $30.7 \%$ & $19.0 \%$ & $29.1 \%$ \\
\hline \multirow{2}{*}{ Excelente } & 19 & 1 & 20 \\
\hline & $15.0 \%$ & $4.8 \%$ & $13.5 \%$ \\
\hline \multirow{2}{*}{ Total } & 127 & 21 & 148 \\
\hline & $100.0 \%$ & $100.0 \%$ & $100.0 \%$ \\
\hline
\end{tabular}

La tabla 2 presenta los valores de los estadísticos de la prueba que mide la inteligencia emocional-social en los dos grupos en los que se dividió la muestra, tanto en la escala total como en las dimensiones o subescalas.

Tabla 2. Estadísticos de la prueba de inteligencia emocional-social y sus dimensiones según frecuencia de asistencia a misa.

\begin{tabular}{|c|c|c|c|c|}
\hline \multirow{3}{*}{$\begin{array}{l}\text { Dimensiones o } \\
\text { Sub - escalas }\end{array}$} & \multicolumn{4}{|c|}{ Frecuencia de asistencia a Misa } \\
\hline & \multicolumn{2}{|c|}{$\begin{array}{c}\text { Católico practicante } \\
\qquad n=127\end{array}$} & \multicolumn{2}{|c|}{$\begin{array}{l}\text { Católico NO practicante } \\
\qquad n=21\end{array}$} \\
\hline & Promedio & $\begin{array}{l}\text { Desviación } \\
\text { estándar }\end{array}$ & Promedio & $\begin{array}{l}\text { Desviación } \\
\text { estándar }\end{array}$ \\
\hline - Intrapersonal & 14.49 & 4.180 & 13.33 & 4.017 \\
\hline - Interpersonal & 39.83 & 4.088 & 37.10 & 4.949 \\
\hline $\begin{array}{l}\text { - Manejo del } \\
\text { estrés }\end{array}$ & 33.52 & 6.894 & 29.90 & 7.259 \\
\hline - Adaptabilidad & 29.33 & 4.424 & 29.81 & 5.115 \\
\hline $\begin{array}{l}\text { - Estado de ánimo } \\
\text { general }\end{array}$ & 46.06 & 6.064 & 43.57 & 8.687 \\
\hline $\begin{array}{l}\text { Inteligencia } \\
\text { Emocional - Social }\end{array}$ & 117.17 & 11.914 & 110.14 & 12.022 \\
\hline
\end{tabular}


La tabla 3 indica los valores obtenidos con la prueba t de Student, mostrando solo las subescalas o dimensiones donde se encontraron diferencias significativas entre los dos grupos (católicos practicantes y no practicantes). También se muestran los valores de la escala total porque se encontraron diferencias significativas. Se trabajó con un nivel de significación de .05.

Tabla 3. Prueba t de Student de la Inteligencia emocional-social y sus dimensiones entre católicos practicantes y no practicantes.

\begin{tabular}{lccc}
\hline \multicolumn{1}{c}{ Subescalas } & $\mathbf{t}$ & gl & Sig. (bilateral) \\
\hline - Interpersonal & 2.758 & 146 & .007 \\
- Manejo del estrés & 2.210 & 146 & .029 \\
\hline Inteligencia emocional -Social & 2.502 & 146 & .013 \\
\hline
\end{tabular}

\section{DISCUSIÓN}

En la presente investigación se encontró que una espiritualidad más desarrollada lleva también a mejores niveles de inteligencia emocional-social, como se muestra en la tabla 1. En ella se observa que los estudiantes católicos practicantes alcanzan mayores niveles de inteligencia emocional-social (IES) y en mayor número que los estudiantes católicos no practicantes. Debido a que los dos grupos tienen tamaños diferentes, se calculó los porcentajes de estudiantes que se encuentran en cada nivel de desarrollo de IES tanto en el grupo de católicos practicantes como no practicantes, y se halló que a mayor frecuencia de asistencia a misa, mayor nivel de desarrollo de inteligencia, emocional-social. Ya que como se estableció para esta investigación se considera como católico practicante a los estudiantes que asisten a Misa como mínimo cada 2 meses y como católico no practicante a los estudiantes que asisten a misa cada 3 meses o en forma más distanciada en el tiempo.

En la tabla 1 se observa que mientras el grupo de estudiantes católicos practicantes alcanza niveles de desarrollo de IES excelentes (15\%) y muy altos $(30.7 \%)$, el grupo de no practicantes apenas llega en $4.8 \%$ al nivel excelente y en $19 \%$ al nivel muy alto. En el nivel alto encontramos que el grupo católico practicante alcanza un $26 \%$ frente al $23.8 \%$ del otro grupo. En el nivel medio encontramos que el grupo católico practicante presenta un $27.6 \%$ mientras el grupo católico no practicante presenta en este nivel su mayor porcentaje, con $47.6 \%$; aquí observamos que mientras el mayor porcentaje de estudiantes del grupo católico no practicante se concentra en el nivel medio de IES, en el grupo católico practicante el mayor porcentaje de estudiantes se concentra en el nivel muy alto de IES. Finalmente, en el nivel bajo o por debajo del promedio de IES, encontramos solo un $0.8 \%$ del grupo católico practicante, mientras que del grupo católico no practicante encontramos a un $4.8 \%$ de estos estudiantes.

Debe notarse la diferencia encontrada en la medición de la variable entre los dos grupos, se observa que a pesar de que los dos grupos son católicos, hay una gran 
diferencia entre un católico practicante y uno no practicante. Se hace la indicación que, en la actualidad, en la que sufrimos un relativismo exagerado, mucha gente se declara católica pero ignora lo que significa ser realmente católico o quiere acomodar la doctrina católica a lo que le gustaría que fuera, para que vaya de acuerdo a sus propios intereses. Pero un católico practicante o verdadero cultiva su espiritualidad siguiendo lo que le indica su Iglesia (pues esta última recoge las enseñanzas de Jesucristo) y frecuentando los sacramentos (confesión, comunión, etc.) que también fueron instituidos por el mismo Jesucristo. Existen personas que creen que ya son católicas porque alguna vez fueron bautizadas o porque se declaran así sin ningún fundamento, y despotrican contra la Iglesia Católica y sus representantes, como su santidad el papa, o nuestro cardenal Juan Luis Cipriani. El bautismo es un sacramento de iniciación, pero tenemos que seguir cultivando nuestra espiritualidad. Por ejemplo: una persona que se bautiza en la religión católica y que no sigue su doctrina es como aquella persona que ingresa a la universidad (está iniciando algo) pero jamás estudia, no se preocupa por su formación profesional y ni siquiera asiste a clases pero al cabo de cinco años pretende que le otorguen el título profesional porque cree estar preparado para ejercer la profesión y además se queja de su universidad diciendo que es tan mala que no aprendió nada en ella.

Debemos procurar que la espiritualidad se convierta en el camino a seguir para alcanzar a Dios, de acuerdo a la huella que el mismo Dios deja en el alma de la persona (Sánchez Griese, 2007) citado por Salgado (2012). Esta verdad fue resumida hace muchos siglos (año 397 d.C.) por un patriarca de la Iglesia Católica, San Agustín, quien escribió en su libro Confesiones: "Nos has hecho para Ti y nuestro corazón está inquieto mientras no descansa en Ti".

Finalmente, en la tabla 2 se observa que los puntajes promedio obtenidos en las diferentes subescalas y en la escala total de inteligencia emocional-social son mayores en el grupo de estudiantes católicos practicantes que en el de no practicantes. La prueba t de Student tuvo como resultado que estas diferencias entre los dos grupos son significativas en las dimensiones o subescalas interpersonal $(p=.007)$, manejo del estrés ( $p=.029$ ) y en la escala total de inteligencia emocional-social $(p=.013)$, como se observa en la tabla 3. Por lo anterior, se acepta la hipótesis.

\section{CONCLUSIONES}

1. Existe diferencia significativa entre los estudiantes católicos practicantes y no practicantes con respecto a la inteligencia emocional-social y a sus dimensiones o subescalas interpersonal y manejo del estrés, a favor de los católicos practicantes.

2. La vida espiritual es muy importante para el ser humano, a tal punto que su desarrollo lo humaniza y contribuye al progreso de sus otras áreas.

3. La asistencia a misa desarrolla y mejora la vida espiritual de la persona, y, en consecuencia, su inteligencia emocional-social.

4. La asistencia a misa es importante para el desarrollo y progreso de la inteligencia emocional-social, especialmente en las subescalas interpersonal y manejo del estrés. 


\section{REFERENCIAS BIBLIOGRÁFICAS}

Bar-On, R. (2006). The Bar-On model of emotional-social intelligence (ESI) [EI modelo de Bar-On de inteligencia emocional-social (IES)]. Psicothema, 18, 13-25.

Catecismo Menor. (2012). Lima, Perú: Arzobispado de Lima.

Escurra, L. y Delgado, A. (2001). Inteligencia emocional y necesidad cognitiva en estudiantes universitarios. Revista de investigación en Psicología (IIPSI), 4 (2), 83-96.

Ferrándiz, C., Hernández, D., Bermejo, R., Ferrando, M. y Sáinz, M. (2012). La inteligencia emocional y social en la niñez y adolescencia: validación castellana de un instrumento para su medida. Revista de Psicodidáctica, 17 (2), 309-339.

Fischman, D. (2014). Inteligencia emocional vs. Inteligencia espiritual. Recuperado de la página Web de David Fischman - Artículos. http://www.davidfischman.com/ articulos/detalle.php?lang=es\&id=116

Molero, C., Saíz, E. y Esteban, C. (1998). Revisión histórica del concepto de inteligencia: una aproximación a la inteligencia emocional. Revista Latinoamericana de Psicología, 30(1), 11-30.

Pérez, L. y Beltrán, J. (2006). Dos décadas de “inteligencias múltiples": implicaciones para la psicología de la educación. Papeles del psicólogo, 27(3), 147-164.

Ugarriza, N. (2001). La evaluación de la inteligencia emocional a través del inventario de Bar-On (I-CE). en una muestra de Lima Metropolitana. Persona, 4, 129-160.

Ugarriza, N. y Pajares, L. (2005). Adaptación y estandarización del inventario de inteligencia emocional de BarOn ICE: NA, en niños yadolescentes. Manual técnico. Lima, Perú.

Salgado, A. (2012). Efectos del bienestar espiritual sobre la resiliencia en estudiantes universitarios de Argentina, Bolivia, Perú y República Dominicana. (Tesis de doctorado). Universidad Nacional Mayor de San Marcos, Lima - Perú.

S.S. BenedictoXVI. (2007). Pensamientos espirituales (Abril 2005-Marzo 2006). Bogotá, Colombia: San Pablo. 